\title{
Supervivencia de mujeres jóvenes con cáncer de mama (CaMa) en el Instituto de Cancerología (IDC) Las Américas (Medellín) entre 2007 y 2016
}

- María Elvira Montoya, Luis Rodolfo Gómez, Héctor Iván García, Mileny Janeth Castaño

Instituto de Cancerología (Medellín, Antioquia)

Introducción: la incidencia de CaMa en jóvenes latinoamericanas es del 20\%, en Colombia fluctúa entre el $10 \%$ y $24 \%$. Su gravedad se atribuye al diagnóstico en estadios avanzados, mayor frecuencia de tumores triple negativos y HER2+. El objetivo fue describir la supervivencia global (SG) de mujeres $\leq 40$ años con CaMa infiltrante operadas en el IDC entre 2007 y 2016.

Materiales y métodos: estudio de seguimiento de una cohorte retrospectiva con datos del registro institucional. La supervivencia se calculó desde el diagnóstico hasta la fecha del último control médico o muerte. Se elaboraron curvas de supervivencia de Kaplan-Meier y se compararon con el logrank test.

Resultados: en el período se operaron 4.425 mujeres, de las cuales $374(8,5 \%)$ tenían $\leq 40$ años. Al diagnóstico 64 (17,1\%) mujeres eran estadio I; 186 (49,7\%), estadio II; 118 (31,5\%), estadio III; y 6 (1,6\%), estadio IV. Por subtipos moleculares hubo 90 (26\%) luminal A, 129 (37,3\%) luminal B HER2-, 71 (20,5\%) luminal B HER2+, 19 (5,5\%) HER2- enriquecido y 37 (10,7\%) triple negativo. Se encontraron 94 (26\%) pacientes HER2+. Tuvieron recaída locorregional 27 (7,2\%) pacientes y 78 (20,9\%) desarrollaron metástasis, especialmente al sistema óseo 48 (12,8\%). La mediana de seguimiento fue 45 meses, con una SG del 86,2\% (IC95\% 81,3-89,9). La SG del estadio I fue del 100\%; estadio II, 91,2\%; estadio III, 77,1\%; y estadio IV, 50\% ( $p<0,05)$. La supervivencia de tumores triple negativos fue la más baja, 72,1 (IC95\% 52,8-84,6).

Conclusiones: la SG fue similar a las reportadas por otros estudios y fue menor en estadios avanzados y tumores triple negativos. 